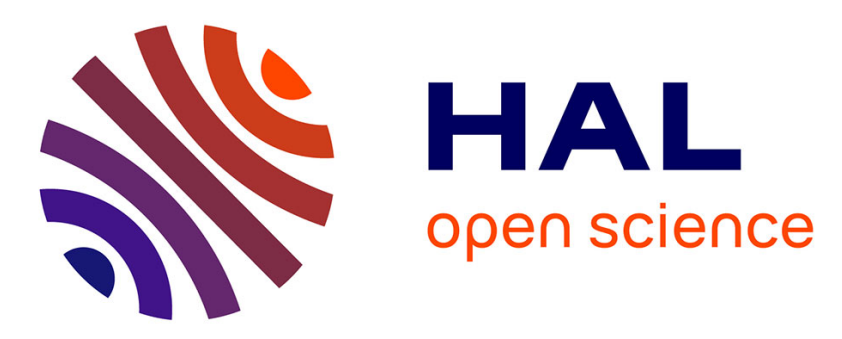

\title{
Zero-Emission Vehicles Sonification Strategy Based on Shepard-Risset Glissando
}

\author{
Sebastien Denjean, Richard Kronland-Martinet, Vincent Roussarie, Sølvi \\ Ystad
}

\section{- To cite this version:}

Sebastien Denjean, Richard Kronland-Martinet, Vincent Roussarie, Sølvi Ystad. Zero-Emission Vehicles Sonification Strategy Based on Shepard-Risset Glissando. 14th International Symposium on Computer Music Multidisciplinary Research, Oct 2019, Marseille, France. hal-02429934

\section{HAL Id: hal-02429934 \\ https://hal.science/hal-02429934}

Submitted on 7 Jan 2020

HAL is a multi-disciplinary open access archive for the deposit and dissemination of scientific research documents, whether they are published or not. The documents may come from teaching and research institutions in France or abroad, or from public or private research centers.
L'archive ouverte pluridisciplinaire HAL, est destinée au dépôt et à la diffusion de documents scientifiques de niveau recherche, publiés ou non, émanant des établissements d'enseignement et de recherche français ou étrangers, des laboratoires publics ou privés. 


\title{
Zero-Emission Vehicles Sonification Strategy Based on Shepard-Risset Glissando
}

\author{
Sebastien DENJEAN ${ }^{1,2}$, Richard KRONLAND-MARTINET ${ }^{2}$, Vincent \\ ROUSSARIE $^{1}$ and Sølvi YSTAD ${ }^{2}$, \\ ${ }^{1}$ Groupe PSA, Scientific Department and Disruptive Technologies, 78943 Vélizy- \\ Villacoublay CEDEX, France \\ ${ }^{2}$ Aix-Marseille Univ, CNRS, PRISM - UMR 7061, Marseille, France \\ sebastien.denjean@mpsa.com
}

\begin{abstract}
In this paper we present a sonification strategy developed for electric vehicles aiming to synthetize a new engine sound to enhance the driver's dynamic perception of his vehicle. We chose to mimic the internal combustion engine (ICE) noise by informing the driver through pitch variations. However, ICE noise pitch variations are correlated to the engine's rotations per minute (RPM) and its dynamics is covered within a limited vehicle speed range. In order to inform the driver with a significant pitch variation throughout the full vehicle speed range, we based our sonification strategy on the Shepard-Risset glissando. These illusory infinite ascending/descending sounds enable to represent accelerations with significant pitch variations for an unlimited range of speeds. In a way, we stay within the metaphor of ICE noise with unheard gearshifts. We tested this sonification strategy in a perceptual test in a driving simulator and showed that the mapping of this acoustical feedback affects the drivers' perception of vehicle dynamics.
\end{abstract}

Keywords: Sonification, automotive acoustics, multisensory perception

\section{Introduction}

With the increasingly tight limits of greenhouse gases emission imposed by governments, car manufacturers need to increase the share of electric motorizations in their series of vehicles. If the development of electric motorizations can help to reduce the in-use emissions of greenhouse gases, these quieter motorizations also lead to a radical change in the acoustic feedback that the driver perceives in the passenger compartment. This reduction of noise level in the passenger compartment has become a strong selling argument for such vehicles. However, although this reduction of noise is believed to be positive for the comfort of passenger, it can also become problematic in some cases. At low speeds for instance, when wind and rolling noises are very low, the loss of Internal Combustion Engine (ICE) feedback makes electric vehicles inaudible for pedestrians and can lead to dangerous situations [1-4]. Standards are now imposed to car manufacturers to equip their vehicles with Acoustic Vehicle Alert System (AVAS) to generate sounds that enable pedestrians to hear their vehicles. 
In addition to the loss of informative noise outside of the vehicle, the loss of ICE noise is also an issue for the driver. ICE noise is indeed the most significant automotive noise source. On the one hand, it has a strong emotional power, being the main vector of evoked sportiness or powerfulness of the vehicle. Its loss can thus have a negative impact on driving pleasure and on brand identity. On the other hand, ICE noise is also the main acoustic information related to the vehicle dynamics that may influence driver's speed perception. Horswill and Plooy (2008) indeed showed that drivers underestimate their speed with a reduced acoustic feedback loudness. In a previous study, we also showed that acoustic feedback affects drivers' perception of acceleration (Denjean et al., 2013). The loss of ICE can thus affect the drivers' perception of both vehicle speed and acceleration.

With the development of electric motorizations, we loose a significant part of the dynamic information usually conveyed by ICE noise while conserving the disturbance due to wind and rolling noises. In a way, electric vehicles are not vehicles without noise but vehicles with only noise. We developed a sonification strategy to synthetize a new engine sound for electric vehicles to give back the information conveyed by engine noise in ICE cars.

\section{Sonification Strategy}

With the freedom of synthesized sounds in sound design, sonification of quiet(er) vehicles is a great opportunity to shape the soundscape of the vehicle. Sounds can give character to the vehicle, with a technological or even futuristic dimension. Sonification is a key for car manufacturers to work on its acoustic brand identity, and a tool to work on perceived quality of the cab acoustics. Sonification can also be used to inform the driver.

In this work, we focused on the dynamic feedback, setting esthetical design aside as a first step. We wanted this sound feedback to be able to inform the driver on the vehicle dynamics regardless of its design, and thus we worked on the invariants of sound dynamism. We chose to build our sonification strategy by mimicking ICE noise to achieve a natural and intuitive way of informing the drivers.

\subsection{Objectives}

We wanted our sonification strategy to convey information through the same levers as ICE noise, but with its own 'electric' personality. In a way, we wanted this sound to speak the same language as the ICE, but through a different voice.

Similarly, to ICE noise, we decided to inform the driver through pitch variations. However, pitch variations from ICE noise are correlated to the rotations per minute (RPM) of the engine and its dynamics is covered in a limited range of the vehicle speed. During acceleration, the pitch grows up to the high RPM of a given gear ratio before falling down at each gearshift. Thanks to this pitch decrease of each gear shift, the ICE pitch range is quite limited over the whole vehicle speed range. However, in electric vehicles, there are no gear shifts, and pitch range is therefore much bigger, since it is covered by only one gear. Even if we decided to simulate non-physical 
acoustical gearshifts in our sonification strategy, we still would have to address the issue of the pitch dynamics in our sound. In ICE vehicle, the pitch of the engine noise grows around 2.5 octaves during one gearshift. It would not be possible to reproduce this pitch increase 5 times within the whole vehicle speed range. The sounds would simply be too high-pitched at high speeds. Even if we adapted the pitch range to fulfill the whole vehicle speed range, we would face too low pitch variations for low accelerations and provide information to the driver that would not be sufficiently precise to estimate his acceleration.

Sound synthesis was the solution to this issue, and an interesting answer to the problem was the Shepard-Risset glissando illusion with continually ascending or descending pitch. It could produce high pitch variations to precisely inform even for low vehicle accelerations while keeping a constrained pitch for the whole speed range of the vehicle. In a way, this illusion enabled us to conserve the metaphor of an ICE noise even with unheard gearshifts.

\subsection{Shepard-Risset Glissando Based Sonification Strategy}

Principle Shepard made tones that created the illusion of a musical scale that continuously ascends or descends in pitch [7]. These tones are composed of a superposition of sine waves separated by octaves with a loudness controlled by a raised cosine function. Low-pitched and high-pitched components are almost inaudible, while central components are clearly audible. These overlapping components are exactly one octave apart, and each scale fades in and out so that hearing the beginning or end of any given scale is impossible. When ascending this scale composed of twelve tones, we can hear the pitch growing at each tone. At the thirteenth tone, the first tone of the scale is heard again, but we still have the sensation of a growing pitch thanks to the specific construction of these tones. Risset used this illusion to create a glissando based on this scale, by simulating continuous transitions from one tone to the other.

We adapted this illusion to our sonification application. We chose to reduce the bandwidth of the raised cosine window to keep a limited range of octaves played at once. This modification allowed us to propose an additional control through the shifting of this window to change pitch through the spectral centroid of the generated sounds. To enhance the perceived illusion and open new design possibilities, we also chose to play chords instead of tones.

Synthesizer development We developed a synthesizer in Max/MSP allowing us to create these sounds and map our feedback to the vehicle speed and acceleration in real-time. Each parameter of the sine wave components can hereby be tuned and the raised cosine function can be used to define the loudness window.

Harmonic comb The user can choose the components of the chord selecting the tones on the musical scale. This leads to the creation of a harmonic comb composed by sine waves corresponding to these tones and duplicated at each octave. The frequencies of the components of this harmonic comb are controlled by a saw tooth function that browse the scale periodically. The amplitude of each component is defined by a loudness window. 
Loudness window The user can define the bandwidth and the central frequency of the loudness window. This window shape responds to a raised cosine function and defines the amplitude of each harmonic component of the comb.

Mapping We mapped the pitch of the generated sounds to the vehicle speed. We chose a generic linear mapping between pitch and vehicle speed $\left(S_{v}\right)$ based on the power law:

$$
\text { Pitch }=\mathrm{kS}_{\mathrm{v}}+\mathrm{c}
$$

With the specific construction of our sound feedback, we have two independent ways to vary the pitch of the generated sounds: with the center frequency of the loudness window $\left(\mathrm{F}_{\mathrm{c}}\right)$ and the sweeping speed $\left(\mathrm{S}_{\mathrm{s}}\right)$ of the harmonic comb (the frequency of the saw tooth function). We declined our mapping to these two parameters, linking the logarithm of the center frequency of the window to vehicle speed, and deriving this formula to map the harmonic comb swiping speed to vehicle speed $\left(S_{v}\right)$ and acceleration $\left(A_{v}\right)$.

$$
\begin{gathered}
\log \left(\mathrm{F}_{\mathrm{c}}\right)=\mathrm{k}_{1} \mathrm{~S}_{\mathrm{v}}+\mathrm{c}_{1} \\
\mathrm{~S}_{\mathrm{s}}=\mathrm{k}_{2} \mathrm{~A}_{\mathrm{v}}
\end{gathered}
$$

Both controls induce pitch variations of the generated sounds and informs the driver about the vehicle speed (with the spectral centroid of the loudness window) and the vehicle acceleration (through the swiping speed of the harmonic comb).

We tested this sonification strategy in a perceptual test in a driving simulator to evaluate the impact of this mapping on the drivers' perception of vehicle dynamics.

\section{Impact of Shepard-Risset Based Sonification Strategy on Drivers' Perception of Vehicle Speed and Acceleration}

In the previous chapter we developed the sonification strategy to provide dynamic information through acoustic feedback to the driver. We focused our strategy on acoustic feedback on speed and acceleration, which is the main information used while driving and usually brought by ICE noise in cars with combustion engines. To evaluate the impact of the proposed sonification strategy on drivers' perception, we ran a perceptual test in a driving simulator.

\subsection{Experiment Objectives}

In a previous study we showed that the sound feedback plays a role in multisensory speed perception among drivers (...). The main goal of this experiment was to evaluate the impact of the sonification strategy on the drivers' speed perception.

However, speed also strongly depends on the preceding acceleration ((Salvatore, 1967; Recarte and Nunes, 1996). We designed our sonification strategy relying on 
this information. The proposed sound, based on the Shepard-Risset glissando, is well suited to give strong acceleration information with the variation of pitch, but can give fuzzy information about absolute speed with its paradoxical pitch, even if we correlate the spectral centroid to vehicle speed.

We assumed that the sonification mapping would affect the drivers' perceived acceleration, and thus their speed production. The quicker the pitch variation the stronger the drivers perceived acceleration, inciting them to produce slower speeds.

To validate this assumption, we focused on the mapping between the swiping speed of the harmonic comb and the vehicle acceleration. We fixed the mapping between the filtering window displacement and the speed, and tested different mappings of this harmonic comb swiping speed according to acceleration.

We asked participants to accelerate or decelerate without the speedometer information to a provide a target speed in different sound conditions.

\subsection{Method}

\section{Participants}

29 volunteers (3 women and 26 men) employees of the PSA group, participated in this study. They all held a valid driver license and declared to have normal or corrected to normal vision and normal audition.

\section{Experimental Device}

We chose to run this perceptual experiment on a driving simulator to control the stimuli presented to the participants and ensure its repeatability. For this experiment we used the fixed-based driving simulator of the PSA group. This driving simulator is composed of the front half of a car disposed in front of a hemi-cylindrical screen. The scene used for the simulation represented a straight two-lane urban road. A picture of the driving simulator and an extraction of the scene are presented Figure 1.
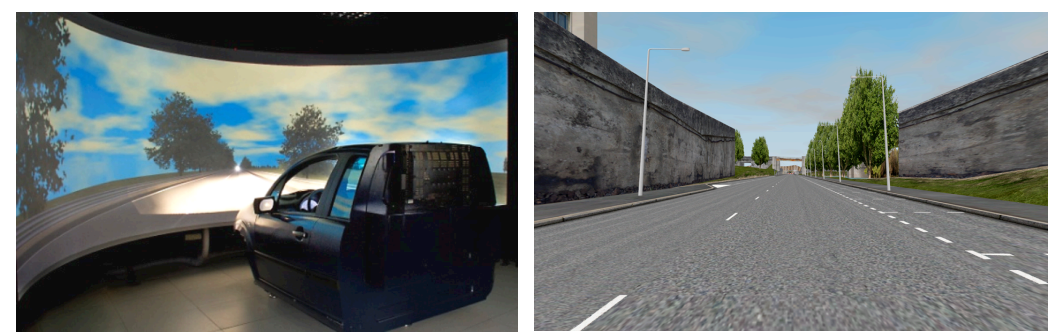

Fig. 1. Picture of the driving simulator and the driving scene used for the perceptual study

The vehicle noise presented to the participants in the driving simulator was based on recordings in an actual car with a binaural dummy head. They were replayed in granular synthesis and our sonification proposals were mixed with this noise. This global mix was played back in the simulator thanks to a five loudspeaker sound system. 


\section{Experimental Variables}

Vehicle speed and acceleration Participants had to reach 2 target speeds, 50 and 70 $\mathrm{km} / \mathrm{h}$, either accelerating from $30 \mathrm{~km} / \mathrm{h}$ below the target (acceleration condition) or decelerating from $30 \mathrm{~km} / \mathrm{h}$ above the target (deceleration condition). The acceleration was controlled and fixed to 2 values in the acceleration and deceleration conditions: $+/-0.75 \mathrm{~m} / \mathrm{s}^{2}$ or $+/-1.5 \mathrm{~m} / \mathrm{s}^{2}$. We thus tested 4 acceleration and 4 deceleration conditions.

Sonification parameters To focus on the impact of the harmonic comb speed variation on the perceived dynamics, all other sonification parameters were fixed. We used a major triad (tonal, major third and fifth) duplicated to other octaves as a basis for the sound feedback. This harmonic comb had been filtered with a 4 octave length window with a central frequency varying exponentially with vehicle speed from 120 $\mathrm{Hz}$ at idle to $660 \mathrm{~Hz}$ at $130 \mathrm{~km} / \mathrm{h}$ (approximately 1 octave every $55 \mathrm{~km} / \mathrm{h}$ ).

For the mapping between swiping speed of the harmonic comb and the vehicle acceleration, we tested 3 linear mappings with constant $\mathrm{k}_{2}=0.04,0.08$ and 0.16 . For the rest of the article, we will use the mapping with a constant $\mathrm{k}_{2}=0.08$ as reference and refer to the mappings as mapping $0.5\left(\mathrm{k}_{2}=0.04\right)$, mapping $1\left(\mathrm{k}_{2}=0.08\right)$ and mapping $2\left(\mathrm{k}_{2}=0.16\right)$. It is interesting to notice that we will encounter situations with the same sound feedback at different visual speeds, with mapping 1 at $0.75 \mathrm{~m} / \mathrm{s}^{2}$ and mapping 0.5 at $1.5 \mathrm{~m} / \mathrm{s}^{2}$, or mapping 1 at $1.5 \mathrm{~m} / \mathrm{s}^{2}$ and mapping 2 at $0.75 \mathrm{~m} / \mathrm{s}^{2}$.

We added a control condition without sonification to the 3 previous conditions.

Experimental variables summary These experimental variables that are summed up in the table have been crossed into a full factorial design. Each stimulus has been repeated 3 times, leading to an experimental design of 96 stimuli.

Table 1. Experimental variable summary. All variables were crossed in a complete experimental design and each stimulus was repeated 3 times leading to 96 stimuli by participant

\begin{tabular}{cccc}
\hline Use case & Target speed & Acceleration level & Sound feedback \\
\hline Acceleration & $50 \mathrm{~km} / \mathrm{h}$ & $+/-0.75 \mathrm{~m} / \mathrm{s}^{2}$ & $\begin{array}{c}\text { Mapping 0.5 }\left(\mathrm{k}_{2}=0.04\right) \\
\text { Mapping } 1\left(\mathrm{k}_{2}=0.08\right)\end{array}$ \\
Deceleration & $70 \mathrm{~km} / \mathrm{h}$ & $+/-1.5 \mathrm{~m} / \mathrm{s}^{2}$ & $\begin{array}{c}\left.\text { Mapping 2 ( } \mathrm{k}_{2}=0.16\right) \\
\text { Electric vehicle (no sonification) }\end{array}$ \\
\hline
\end{tabular}

\section{Procedure}

Participant tasks Participants had to perform a symmetric tasks during the acceleration and deceleration conditions (Fig. 2).

During acceleration, subjects were launched at a constant speed $(20$ or $40 \mathrm{~km} / \mathrm{h}$ depending on the target speed 50 or $70 \mathrm{~km} / \mathrm{h}$ ). The driving simulation regulated the vehicle speed and the driver did not press the accelerator pedal, as if the subject drove with a speed regulator. When entering the acceleration zone, the subject pressed the accelerator pedal to accelerate. The vehicle acceleration was regulated by the simulation (at 0.75 or $1.5 \mathrm{~m} / \mathrm{s}^{2}$ ) and the driver had to stop the acceleration by releasing the pressure on the accelerator when he thought he had reached the target speed. After the acceleration, the vehicle speed was kept constant by the driving simulation. 
In the deceleration condition, the task was symmetrical, and the participants were asked to press the accelerator pedal to keep speed constant (at 80 or $100 \mathrm{~km} / \mathrm{h}$ depending on the target speed). When they were ready to start, they reduced the pressure on the accelerator to begin the deceleration (at 0.75 or $1.5 \mathrm{~m} / \mathrm{s}^{2}$ depending on test condition) and had to press the accelerator again when they thought they had reached the target speed.
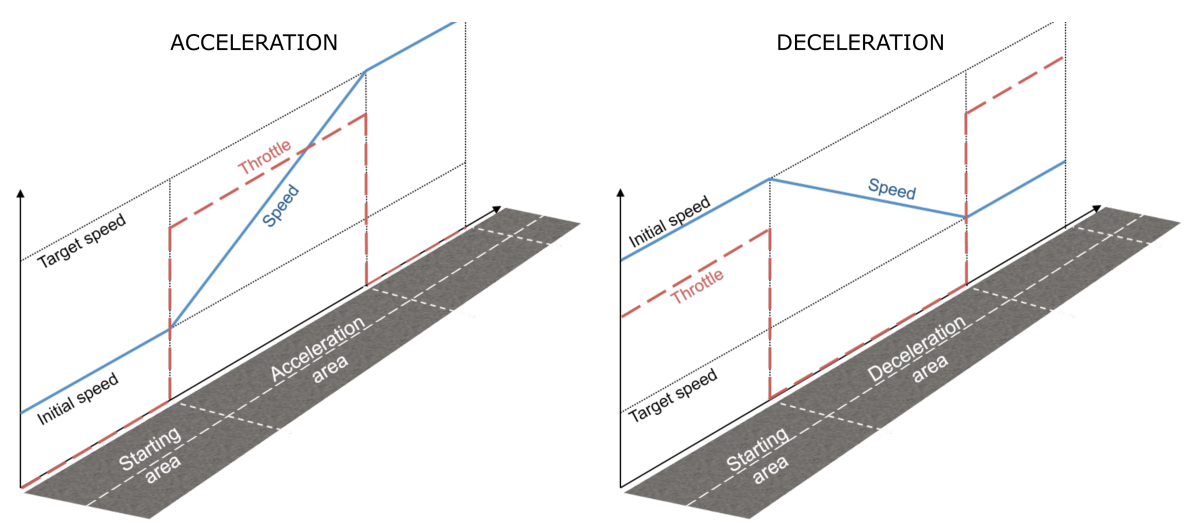

Fig. 2. Test procedure for acceleration and deceleration conditions

Test sessions Stimuli were divided in two blocs by the acceleration/deceleration condition. To avoid learning effects, the order of these sessions were pseudorandomly launched between subjects. The whole test lasted approximately $1 \mathrm{~h} 45$.

At the beginning of the test session, the experimenter presented the experiment to the participant. The experimenter explained that the participant would have to evaluate the vehicle speed without a speedometer feedback at different speeds. He also explained that different acoustic feedbacks would be provided, but without mentioning the nature or the type of control of these sounds.

Before the beginning of the test, the participants underwent a training phase. The first training phase aimed to familiarize the participant with the driving simulator: the participant drove freely in the map used for the test. During this phase, the speedometer was visible to the participant to calibrate his speed perception and no sonification was added. Participants were driving in the sound control condition (electric vehicle). When the participant felt comfortable with the driving simulator, the test began.

Each test bloc (acceleration and deceleration) began with a task-learning phase. The experimenter explained the subject's task and a couple of additional tasks were tested to make sure the participant fully understood the task. Stimuli corresponding to the different acceleration intensities, target speeds and sounds were presented with a pseudo-randomized experimental design based on a latin square to reduce learning effects. 


\subsection{Results}

We recorded the speed reached by the participants after the acceleration/deceleration phase as their perceived target speed. We calculated the error between the speed reached by the driver and the target speed to compare the results between the two target speeds tested.

We ran an Analysis of Variance (ANOVA) on the whole set of data to determine the experiment parameters that had a significant impact on the speed reached by the drivers.

Acceleration condition In the case of acceleration, the ANOVA shows a significant effect of the sound condition $(p<0.001)$. A Scheffé post-hoc test separated these four condition in three groups: mapping 2 (mean error $-1.6 \mathrm{~km} / \mathrm{h}$ ), mapping 1 (mean error $0.1 \mathrm{~km} / \mathrm{h}$ ) and electric condition (mean error $0.8 \mathrm{~km} / \mathrm{h}$ ), mapping 0.5 (mean error 1.1 $\mathrm{km} / \mathrm{h}$ ) and electric condition.

The intensity of the acceleration has also a significant impact on the speed reached by the drivers $(p<0.001)$, with a speed error of $-2.8 \mathrm{~km} / \mathrm{h}$ during acceleration and 3 $\mathrm{km} / \mathrm{h}$ during deceleration.

We can also notice a significant effect of the target speed $(p<0.001)$ with a mean error of $0.9 \mathrm{~km} / \mathrm{h}$ at $50 \mathrm{~km} / \mathrm{h}$ and $-0.7 \mathrm{~km} / \mathrm{h}$ at $70 \mathrm{~km} / \mathrm{h}$.

Repetitions also showed significant effects $(p<0.001)$ with a mean speed error of $0.7 \mathrm{~km} / \mathrm{h}$ for the first repetition, $0 \mathrm{~km} / \mathrm{h}$ for the second and $1 \mathrm{~km} / \mathrm{h}$ for the third repetition.

No interaction between these parameters reached significance level of $5 \%$.

Deceleration condition For the deceleration condition, the ANOVA shows the same significant effects. The sound condition has a significant effect on the speed reached by the drivers $(p<0.001)$ and Scheffé post-hoc test sorts the conditions in three groups: mapping 0.5 (mean error $8.5 \mathrm{~km} / \mathrm{h}$ ), mapping 1 (mean error $10.2 \mathrm{~km} / \mathrm{h}$ ) and electric condition (mean error $10.6 \mathrm{~km} / \mathrm{h}$ ), mapping 2 (mean error $12.4 \mathrm{~km} / \mathrm{h}$ ).

We can also notice a significant effect of the intensity of the acceleration $(p<$ 0.001 ) with lower speeds reached at higher decelerations (mean error of $7.5 \mathrm{~km} / \mathrm{h}$ at $1.5 \mathrm{~m} / \mathrm{s}^{2}$ and $13.3 \mathrm{~km} / \mathrm{h}$ at $-0.75 \mathrm{~m} / \mathrm{s}^{2}$ ).

The target speed has also a significant influence on the speed reached by the participants $(p<0.001)$, with a mean error of $10.8 \mathrm{~km} / \mathrm{h}$ at $50 \mathrm{~km} / \mathrm{h}$ and $10 \mathrm{~km} / \mathrm{h}$ at 70 $\mathrm{km} / \mathrm{h}$.

As for the acceleration condition, repetition has also a significant influence on mean speed errors of $11 \mathrm{~km} / \mathrm{h}$ for first, $10.6 \mathrm{~km} / \mathrm{h}$ for the second and $9.6 \mathrm{~km} / \mathrm{h}$ for the third repetition.

No interaction between these parameters reached significance level of $5 \%$.

\subsection{Discussion}

Speed perception and influence of acceleration In Figure 3 we plotted the mean speeds reached by the participants in the acceleration and deceleration conditions for target speeds of $50 \mathrm{~km} / \mathrm{h}$ and both acceleration intensities 0.75 and $1.5 \mathrm{~m} / \mathrm{s}^{2}$. We can notice a global overproduction of speed by the participants, suggesting that they 
compensated their underestimation of speed by reaching speeds higher than the target. [9]. This underestimation of speed is higher at lower speed, in consistency with the literature [9-12], with a mean speed reached of $56 \mathrm{~km} / \mathrm{h}$ for a target speed of $50 \mathrm{~km} / \mathrm{h}$ and $77 \mathrm{~km} / \mathrm{h}$ for a target speed of $70 \mathrm{~km} / \mathrm{h}$.

The value of speed reached by the participants is also modulated with the intensity and sign of the acceleration.

Regarding the sign of the acceleration, we can see that drivers tend to reach higher speeds during deceleration than acceleration, suggesting that they tend to overestimate their acceleration. This overestimation of acceleration makes them think that they accelerated (resp. decelerated) more than they actually did and stopped the acceleration (resp. deceleration) before they should, inducing them to reach lower (resp. higher) speeds.

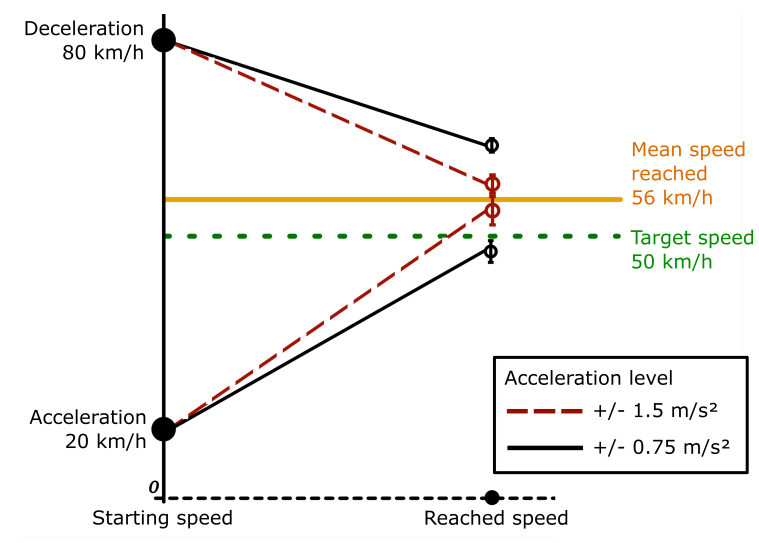

Fig. 3. Mean speeds reached by participants for the target speed of $50 \mathrm{~km} / \mathrm{h}$ at acceleration and deceleration at both acceleration levels.

Regarding the intensity of the acceleration, we can notice that the situation is symmetrical. In the acceleration condition, the drivers tended to reach lower speeds at lower acceleration. Focusing on speed, we can infer that drivers underestimate their speed more after stronger accelerations, consistently with the study of Salvatore [8]. However, during deceleration, the opposite effect can be observed, with lower speeds reached with higher decelerations. This symmetrical result suggest that drivers tend to overestimate their acceleration and even more when its intensity its lower.

This result confirms the major role of acceleration in drivers' speed perception.

Influence of sound feedback Our sonification strategy based on the Shepard-Risset illusion is particularly well suited to inform on vehicle acceleration. More than the association of a pitch to a particular speed, this feedback enables to control the variation of the pitch according to vehicle acceleration.

We assumed that this feedback could modify the perceived acceleration among drivers and modify their speed production. The plot of speeds reached by participants in the different sound conditions confirms this hypothesis. Similarly, to the effect of the intensity of acceleration, we can notice that the effect of sound mapping in symmetrical during acceleration and deceleration. During acceleration, drivers tended 
to reach lower speeds with a sound mapping that had a fast comb swiping. During deceleration, they reached higher speeds with fast comb swiping.

This result suggests that the sound information influenced the perceived acceleration among participants, with a perception of acceleration that increased with the comb swiping.

It is also interesting to observe the control condition without sonification which is included between the conditions with sonification. The comparison with the sonification conditions suggests than we can increase or decrease drivers' perceived acceleration according to the sound mapping.

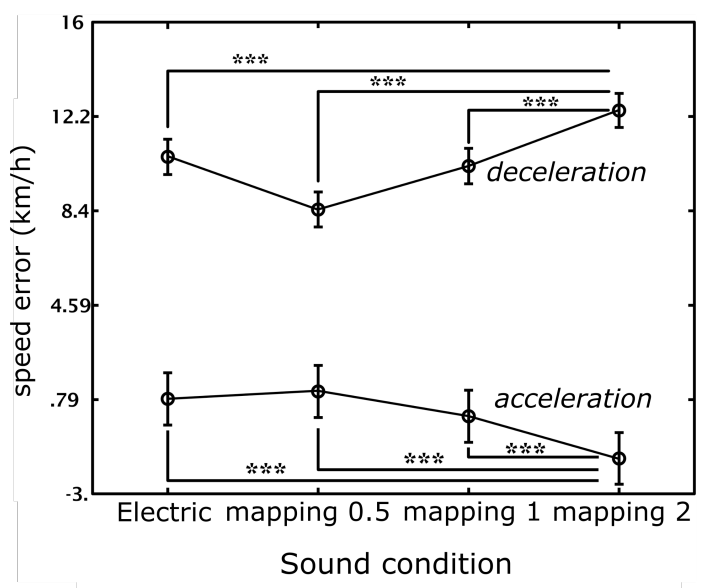

Fig. 4. Errors between speed reached by participants and target speed in acceleration and deceleration in the different sound conditions

Even if this effect is naturally lower than the effect of acceleration intensity, it can contribute to change drivers' acceleration perception. We saw that the drivers tend to overestimate their acceleration and mostly at low acceleration intensities. An exponential mapping can then be used to reduce this overestimation for low accelerations and adjust acceleration perception.

\section{Conclusion}

The development of quieter electric motorizations changes the soundscape perceived by drivers in their vehicles, and this "silence" is often praised as zero emission vehicles (ZEV). However, Internal Combustion Engine (ICE) noise is an important feedback for drivers. It gives precious information about vehicles' dynamics and has an important impact on the emotional level, since it contributes to the evocation of sportiness and fun when driving the vehicle. In this study we developed a sonification strategy to improve the pleasure of the driving experience in electric vehicles. In particular, we wanted the generated engine sound to provide information to the driver and to design the in-cab soundscape according to an emotional state. In this work we focused on the dynamic feedback of the vehicle. We decided to mainly focus on the 
acceleration information, which is of major interest for driving control. The proposed strategy is based on the engine noise metaphor, with a pitch that increases with acceleration, to provide natural and intuitive feedback to the driver. We found that the Shepard-Risset glissando illusion was perfectly suited for this aim. It precisely provided information on the acceleration with fast pitch variations that could be maintained over the whole speed range of the vehicle. We tested different mappings of this sonification strategy with vehicle dynamics and showed that this had an impact on the drivers' acceleration perception and speed production. This sonification strategy can also be tuned to generate more technological sounds than ICE noise and is particularly well suited to answer the issue of interior sonification of zero emission vehicles (ZEV).

\section{Acknowledgements.}

This work was partly supported by the French National Research Agency (ANR-10CORD-0003, MetaSon, "Métaphores sonores", https://metason.prism.cnrs.fr)

\section{References}

1. Garay-Vega, L., Pollard, J. K., Guthy, C., and Hastings, A.: Auditory detectability of hybrid electric vehicles by blind pedestrians. Transportation Research Record : Journal of the Transportation Research Board, 2248(1) :68-73 (2011)

2. Chamard, J.-C. and Roussarie, V.:Design of electric or hybrid vehicle alert sound system for pedestrian. Acoustics 2012 Nantes . (2012).

3. Ashmead, D. H., Grantham, D. W., Maloff, E. S., Hornsby, B., Nakamura, T., Davis, T. J., Pampel, F., and Rushing, E. G.: Auditory perception of motor vehicle travel paths. Human Factors (2012)

4. Altinsoy, E.: The detectability of conventional, hybrid and electric vehicle sounds by sighted, visually impaired and blind pedestrians. In Internoise 2013, Innsbruck (2013)

5. Horswill, M. S. and Plooy, A. M.: Auditory feedback influences perceived driving speeds. Perception, 37(7) :1037. (2008)

6. Denjean, S., Velay, J.-L., Kronland-Martinet, R., Roussarie, V., Sciabica, J.-F., and Ystad, S.:Are electric and hybrid vehicles too quiet for drivers ? In Internoise 2013, Innsbruck (2013)

7. Shepard, R. N.: Circularity in judgments of relative pitch. The Journal of the Acoustical Society of America, 36(12) :2346-2353 (1964)

8. Salvatore, S.: Estimation of vehicular velocity under time limitation and restricted conditions of observation. Technical report, Transportation Research Board (1967)

9. Recarte, M. A. and Nunes, L. M: Perception of speed in an automobile: Estimation and production. Journal of Experimental Psychology: Applied ; Journal of Experimental Psychology: Applied, 2(4) :291 (1996)

10.Evans, L.: Speed estimation from a moving automobile. Ergonomics, 13(2) :219-230 (1970)

11.Miloševic, S.: Perception of vehicle speed. Revija za psihologiju (1986)

12.Recarte, M. A., Conchillo, Á., and Nunes, L. M.: Traffic and transport psychocology, chapter Perception of speed and increments in cars, pages 73-84. Elsevier (2004) 